\title{
Correction of Slice Misalignment in Multi-Breath-Hold Cardiac MRI Scans
}

\author{
Benjamin Villard ${ }^{1}$, Ernesto Zacur ${ }^{1}$, Erica Dall'Armellina ${ }^{2}$, and Vicente Grau ${ }^{1}$ \\ 1 Institute of Biomedical Engineering, University of Oxford, United Kingdom, \\ benjamin.villard@eng.ox.ac.uk, \\ 2 Oxford Acute Vascular Imaging Center, Division of Cardiovascular Medicine, \\ Radcliffe Department of Medicine, University of Oxford
}

\begin{abstract}
Cardiac Magnetic Resonance (CMR) provides unique functional and anatomical visualisation of the macro and micro-structures of the heart. However, CMR acquisition times usually necessitate slices to be acquired at different breath holds, which results in potential misalignment of the acquired slices. Correcting for this spatial misalignment is required for accurate three-dimensional (3D) reconstruction of the heart chambers allowing robust metrics for shape analysis among populations as well as precise representations of individual geometries and scars. While several methods have been proposed to realign slices, their use in other important protocols such as late gadolinium enhancement (LGE) is yet to be demonstrated. We propose a registration framework based on local phase to correct for slice misalignment. Our registration framework is a group registration technique combining long- and short-axis slices. Validation was performed on LGE slices using expert-traced ventricular contours. For 15 clinical multi-breath-hold datasets our method reduced the median discrepancy of moderately misaligned slices from $2.19 \mathrm{~mm}$ to $1.63 \mathrm{~mm}$, and of severely misaligned from $7.33 \mathrm{~mm}$ to $1.96 \mathrm{~mm}$.
\end{abstract}

Keywords: Slice Misalignment, Late Gadolinium Enhancement, CMR

\section{Introduction}

Cardiovascular diseases are one of the world's biggest killers, accounting for over 4 million deaths in Europe yearly [1]. Enabling early diagnosis and effective treatment is essential to the reduction of the burden of cardiovascular diseases.

In recent years much research has looked at creating personalised 3D anatomical models of the heart [2]. These models usually incorporate a geometrical reconstruction of the anatomy in order to understand better cardiovascular functions as well as predict different processes after a clinical event. Also, population studies of cardiac anatomy require precise geometrical reconstructions [3,4]. However the ability to accurately reconstruct heart anatomy from MRI in three dimensions commonly comes with a fundamental challenge: the misalignment between slices acquired at different breath holds. In this paper we discuss an alignment algorithm for individual CMR slices that allows subsequent accurate 
reconstruction of geometrical models of the heart. The algorithm uses the intersection lines between slices, introducing a new cost function designed to be applied on both cine and late gadolinium images, as well as a specifically designed optimisation strategy. The main contributions of this work are:

- The development of a complete framework for the correction of translational and rotational misalignments between CMR slices, based on the combination of short axes (SA) and long axes (LA) images and applicable to cine and LGE CMR scans.

- The introduction of the normalised cross correlation of local phase vectors as a similarity measure that makes the method applicable to different CMR protocols.

- A complete validation using manually traced contours, which includes the estimation of contouring errors.

\subsection{Misalignment between CMR slices}

CMR images allow for detailed ventricular anatomical information as well as an accurate representation of myocardial function by using a plethora of available specialised protocols. Acquiring cardiac images is a complex process due to the constant motion of the heart. Standard clinical protocols do not allow 3D images of the heart in a single acquisition, and thus typically acquire a collection of $2 \mathrm{D}$ slices, oriented either on the short axis or on a long axis plane of the ventricles, each one at a separate breath hold. Electrocardiogram (ECG) allow the images to compensate for cardiac motion. Breath holding at the same lung volume for periods ranging from 7 to 15 seconds is used to reduce slice misalignment from the acquisition. This alignment distortion may be further enhanced by any patient movement inside the scanner, affecting slice spatial coherence as a 3D dataset [5].

\subsection{Motion correction}

A significant amount of research has been dedicated to the correction of CMR slice misalignment. Some studies solely align endo and epicardial contours with regards to each other. This requires the availability of accurate contours, and has the disadvantage of forfeiting all the additional information available in the image values. In practice, due to the inter/intra variability in expert contouring, the final alignment will depend on the expert; on the other hand, if the application requires the smoothest ventricular shapes possible, methods such as [6] can be used. Other studies use volumetric approaches where individual slices are registered to a 3D volume (slice-to-volume registration) [7-9]. The second common approach in the literature uses slice-to-slice alignment (slice-to slice registration), which is also used in our method. This approach is based on using the image intensities at the slice intersections. By optimising over the (dis)similarity between intensities on the intersecting line, optimal alignment can be achieved. Some studies $[10,11]$ use a fixed slice, usually an LA slice, to which all the other slices are aligned to. The drawback to this is the dependence on the choice of the 
reference slice, which can heavily influence the results if a particularly misaligned slice is chosen as the fixed reference. An alternative method is to alternate the reference slice in an iterative manner and allow the other slices to register to it. This has the negative effect of being highly influenced by outliers and makes the process more sensitive to local minima. This results in optimising over a space of $6 n$ parameters, with $n$ being the number of slices, which is costly and less efficient . To minimize the amount of local minima, our method fixes all the other slices and allows only one slice to "move", obtaining the best global alignment. Furthermore using the sum of similarity measures from a slice to several reference slices can lower the influence of outliers, forcing them to converge through an iterative process; also known as alternate optimisation [12]. Theoretically, different alignment strategies could converge to the same minimum, regardless of the optimisation parameters used. However, the presence of local minima means that, in practice, the choice of optimisation parameters has a substantial effect on the result.

\subsection{Similarity function}

Cost functions, otherwise known as similarity measures, provide a measure of (dis)similarity between images/intensities in the domain of image registration. Similarity functions can be feature-based, which aim at the alignment of specific image features (e.g. edges), or voxel-based, which use all intensity values and quantifies their differences. Voxel-based measures can usually be posed in a generative and statistical framework providing a measure of mutual dependence between random variables. The similarity criterion is one of the key factors in the performance of a registration process, and depends on the nature of the data to be registered [13] . Protocols including gadolinium injections suffer from contrast wash-in/wash-out in the images, increasing the disparity in intensities between slices at different time instants. This prevents the use of simple measures based on intensity differences, such as Sum of Absolute/Square Differences (SAD/SSD). Although some studies such as [14] use SSD as a similarity function, they only apply to same plane (2D-2D) registration, whereas in our case, the intensity discrepancy mostly occurs between the LA and SA slices. We con-

sider that the use of a contrast-independent measure based on salient features would be more appropriate to match the line intersection profiles. Phase based metrics have been a popular choice to use in the last years $[15,16]$. Local phase is a contrast independent descriptor of image structure and is thus not affected by intensity discrepancies [17].

\section{Materials and Methods}

\section{$2.1 \quad$ CMR data}

The datasets used consists of DICOM files containing 2D CINE MRI and 2D LGE sequences of size 216 x 256 pixels (approx: $1.41 \mathrm{~mm}$ x $1.41 \mathrm{~mm}$ ). 15 datasets 
(SIEMENS TrioTim 3 Tesla scanner at the John Radcliffe Hospital, Oxford, UK) from different subjects were used. Each dataset contains between 9 and 13 SA slices from apex to base separated by $8 / 10 \mathrm{~mm}$ and $3 \mathrm{LA}$ slices (4 chamber view, 2 chamber view and out-flow tract). For validation purposes, left ventricle epiand endocardial contours were manually traced by an expert on all SA and LA slices using the CMR42 software (Circle Cardiovascular Imaging, Calgary, Canada).

\subsection{Image registration algorithm}

Our method relies on the intensity profiles at the line formed by the intersection between two slices to align the slices together and give spatial coherence to the 3D dataset. This is based on the assumption that two slices will be perfectly aligned when the underlying features of the line profile at their intersection is complementary. Assuming that slices from a subject are triggered at the same cardiac phase the 3D shape/anatomy of the heart remains fixed among slices. Therefore, rigid-body transformations between slices are considered. As such, we perform rigid registration for each of the slices over 6 degrees of freedom of a rigid-body transformation.
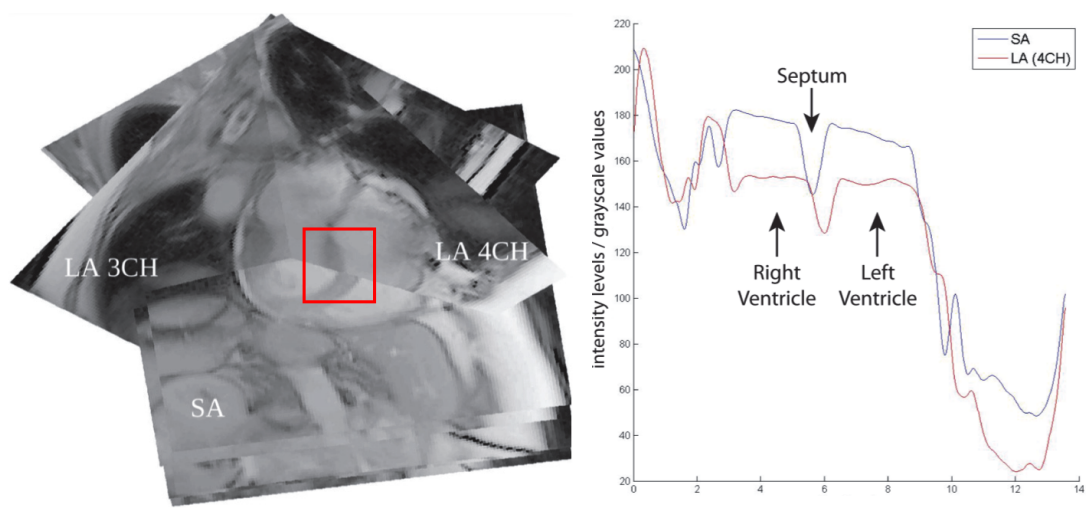

Fig. 1: Left panel shows some 2D MRI slices in their spatial 3D positions, where a clear misalignment can be shown (red box). Right panel shows the intensity profiles along an intersection line.

\subsection{Alignment score and optimisation strategy}

The global motion $(G M)$ discrepancy of the slices can be measured as the sum of (dis)similarity measures $E$ between pair of intersecting slices. Let $S_{i}$ be the $i$-th slice and $\Theta_{i}$ the set of its 6 rigid transformation parameters, for example $\Theta=$ $\left\{t_{x}, t_{y}, t_{z}, \alpha, \beta, \gamma\right\}$ (in our case 3 translations plus 3 Euler angles). Moreover, let $S_{i}^{\Theta_{i}}$ be the transformed version of the slice $S_{i}$ by the rigid transformation defined 
by parameters $\Theta_{i}$. By using this notation, the global motion $(G M)$ discrepancy is given by

$$
G M\left(\Theta_{1}, \Theta_{2}, \ldots, \Theta_{n} ; S_{1}, S_{2}, \ldots, S_{n}\right)=\sum E\left(S_{i}^{\Theta_{i}}, S_{j}^{\Theta_{j}}\right)
$$

where the summation runs over all pairs $S_{i}, S_{j}$ intersecting in a line, i.e. an LA slice will intersect with all the SAs and the other LAs. Minimising $G M$ is akin to finding the parameters for each slice. In this work, this minimisation is performed in an alternate manner, by optimising the parameters $\Theta$ of a single slice whilst leaving the others fixed. As the $G M$ is built as a sum of terms, the iterative minimisation of partial terms results in the minimisation of the global motion discrepancy and the slices match together.

\subsection{Similarity measure}

The particularities of image acquisition described in Section 2.1 prevent the use of simple measures based on intensity differences, such as SAD/SSD. For images with high intensity disparity, feature-based similarity measure can provide a more robust method of assessing similarity between the images at the intersection profiles. As local phase is independent of contrast and not affected by intensity inconsistencies, it is a sensible choice as a similarity measure. The local phase can be obtained through the analytic signal in $1 \mathrm{D}$, and general extensions to higher dimensions have been proposed including the use of oriented filters or the monogenic signal [16]. Although our registration is based on the similarity between the 1D intersecting line profiles, we compute the local phase for an entire image, and then obtain the line intersection between two local phase images as it results in less noise in the line profiles [16]. The normalised cross correlation (NCC) between two profiles is used as similarity measure $E$ for an intersecting pair. In $2 \mathrm{D}$, the local frequency information can be obtained by convolving the images with banks of quadrature pairs of log-Gabor filters [15]. A quadrature filter is a complex valued function which transforms a real valued signal to an analytical signal with weighted frequency components. Convolving the image with a filter will result in response vectors encoding phase and amplitude. By using quadrature filters, local phase $(\Phi)$ can be estimated by

$$
\Phi=\arctan \left(\frac{I_{q}}{I_{p}}\right)
$$

where $I_{q}$ represents the magnitude of the odd filters convolved with the image, and $I_{p}$ the filter response. With respect to $2 \mathrm{D}$, quadrature filters can be generalised through directional formulation [18]. This results in the image that can be seen in Figure 3. Once the local phase images are obtained, the line intersection at both images is taken to account for the similarity measure, using NCC.

\subsection{Contour-based alignment}

Due to inconsistencies in manual contouring of the SA and LA slices, a "perfect" alignment (i.e. one in which the distances between SA and LA contour is zero) 
might not be achievable. In order to establish a baseline error value, we applied a misalignment correction algorithm using the contours from manual images, substituting function $\mathrm{E}($ ) in equation (1) by the Euclidean distance between SA and LA contours. We refer to this algorithm as "contour-based" to differentiate it from the "image-based" algorithm we propose here.

\section{Results}

We investigated the performance of the registration algorithm using the normalised correlation of the local phase signals. The algorithm was used on 15 datasets, each containing 3 LAs and several SAs. The algorithm was run for 9 iterations, for each dataset, in order to evaluate the convergence of the registration. This number was chosen empirically, as the algorithm appeared to converge by then (see Figure 2).

Table 1 shows the mean, median, and standard deviation resulting from contour to contour distance calculations before the alignment and after, using the image based method, and contour only method. The upper part of the table represents all of the 682 individual contour to contour distance for all the datasets. The lower part reports the values for the 82 contours that were deemed significantly misaligned $(>5 \mathrm{~mm})$.

\begin{tabular}{||l|c|c|c||}
\hline & Before & $\begin{array}{l}\text { Image } \\
\text { based }\end{array}$ & $\begin{array}{c}\text { Contour } \\
\text { based }\end{array}$ \\
\hline \hline Median : & 2.19 & 1.63 & 0.31 \\
Mean : & 2.82 & 2.03 & 0.46 \\
Std : & 2.48 & 1.71 & 0.47 \\
\hline Median : & 7.33 & 1.96 & 0.29 \\
Mean : & 7.73 & 2.72 & 0.43 \\
Std : & 2.43 & 2.36 & 0.44 \\
\hline
\end{tabular}

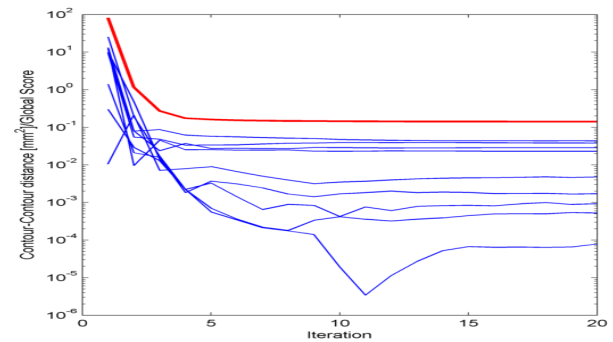

Table 1: Top: LGE phase results for all 15 patients before alignment and after 9 different iterations, as well as with alignment by minimising contours only. Bottom: Same as above but for significantly misaligned slices. Fig. 2: Pairwise energy $E$ between slices over several iterations. Red line shows the global energy $G M$.

\section{Discussion}

Our method relies on using all available information to correct for misalignment by using both the long and short axes slices, in an iterative process. By taking into account all intersections simultaneously, we minimize the effect that individual outliers have on the overall results, forcing these outliers towards the global "consensus" position. By using local phase, we rely on its invariance to changes of intensities in order to focus on obtaining high feature similarity rather than high intensity profile similarity. Relying on the latter assumes that the images to be registered contain similar intensity profiles which is not always the case. 

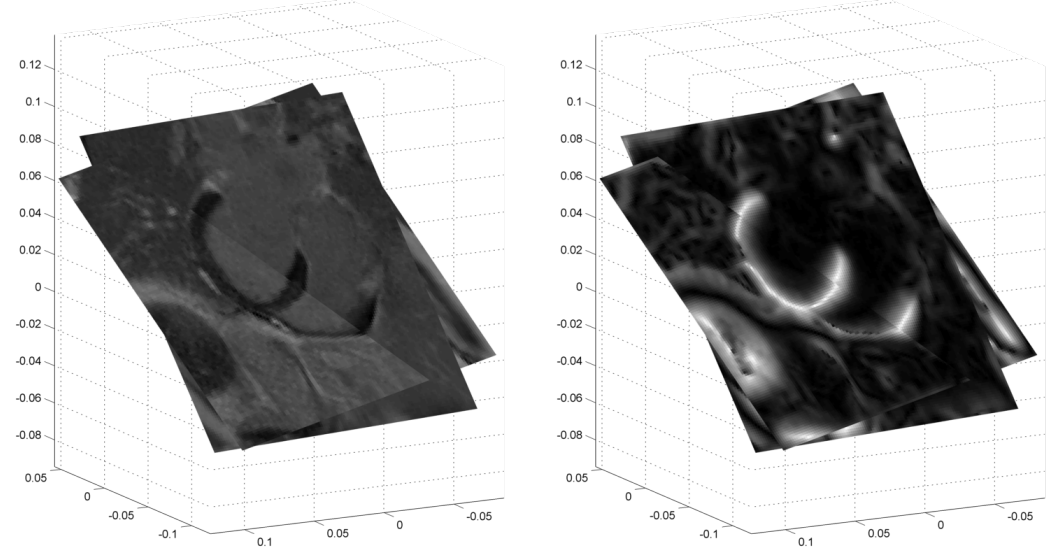

Fig. 3: Late gadolinium image on the left and local phase image on the right

The slice optimisation was constrained to compensate for potential structural symmetry, which might lead to out of plane misalignment such as could be the case with midventricle short axis slices. A hard constraint was chosen as an alternative to introducing a regulariser as the latter would induce a bias towards the initial positions of the planes.

The median and mean values for all 15 patients can be observed to decrease notably, however our method distinguishes itself more with regards to the remarkably misaligned slices. We have defined significantly misaligned slices as slices having higher than $5 \mathrm{~mm}$ between contours. Results show that the median value before the alignment is $7.33 \mathrm{~mm}(7.73 \mathrm{~mm}$ mean $)$. After the alignment, a median of $1.96 \mathrm{~mm}$ was obtained (2.72 $\mathrm{mm}$ mean). By minimising the contour to contour distances only, results show that zero minimal distance is unobtainable, due to the variability in contouring. As such, the lowest median obtainable with our contours was $0.29 \mathrm{~mm}(0.43 \mathrm{~mm}$ mean), which should be accounted for when looking at the image based results.

Oscillations along the iterations of the alternate optimisation are a concern due to the slice dependencies. This can be observed in the behaviour of the global energy at the different iterations (see Figure 2). It can be seen empirically that 10-20 iterations are enough to converge. $G M$ can be seen to decrease and follow a descent path without any jumps occurring, however the evolution of the pairwise energy can result in a non-monotonic (non-descent) path. Even after running the algorithm for more then 100 iterations, some energy pairs will continue oscillating. It can also be seen that for a given pair, the lower the energy, the more it oscillates.

Validation for cardiac image processing comes with some important drawbacks that need to be taken into account. There typically exist no true ground truth due to the nature of the problems. In the majority of cases the ground truth relies on quantification values based on clinical segmentation. However expert 

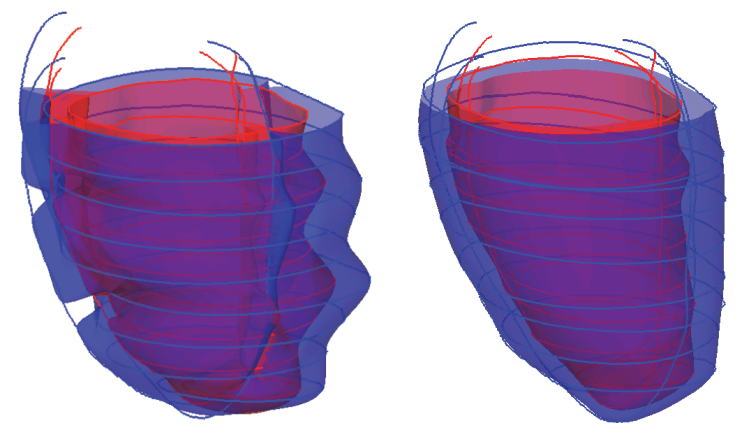

Fig. 4: Surface reconstruction before and after alignment.

segmentation should not be considered as the true ground truth, but more as an approximation, as it suffers from inter-intra expert variability. Furthermore the choice of quantification methods is highly variable and can greatly impact the results.

It can be said that the motion correction algorithm is indispensable for any cardiac anatomical reconstruction which is clearly shown by Figure 4. Several artifacts can be observed such as non aligned SA contours causing a waving surface. Furthermore spatial discrepancies between SAs and LAs produces depth fissures and ridges of the surfaces and discrepancies between LAs do not allow for a good reconstruction of the apical region.

We have presented a phase based registration algorithm that corrects for the misalignment of LGE MRI images. This framework will be used as a preprocessing step in 3D reconstructions of the heart, leading to accurate anatomical models.

\section{Acknowledgments}

BV acknowledges the support of the RCUK Digital Economy Programme grant number EP/G036861/1 (Oxford Centre for Doctoral Training in Healthcare Innovation). EZ acknowledges the Marie Sklodowska-Curie Individual Fellowship from the H2020 EU Framework Programme for Research and Innovation [Proposal No:655020-DTI4micro-MSCA-IF-EF-ST]. ED acknowledges the BHF intermediate clinical research fellow grant (FS/13/71/30378) and the NIHR BRC. VG is supported by a BBSRC grant (BB/I012117/1), an EPSRC grant (EP/J013250/1) and by BHF New Horizon Grant NH/13/30238.

\section{References}

1. escardio.org. European Cardiovascular Disease Statistics 2012, 2012.

2. H.J. Arevalo, F. Vadakkumpadan, E. Guallar, A. Jebb, P. Malamas, K.C. Wu, and N.A. Trayanova. Arrhythmia risk stratification of patients after myocardial infarction using personalized heart models. Nature Communications, 7, 2016. 
3. P. Lamata, M. Sinclair, E. Kerfoot, A. Lee, A. Crozier, B. Blazevic, S. Land, A.J. Lewandowski, D. Barber, and S. Niederer. An automatic service for the personalization of ventricular cardiac meshes. Journal of The Royal Society Interface, 11(91), 2013.

4. A.A. Young and A.F. Frangi. Computational cardiac atlases: from patient to population and back. Experimental Physiology, 94(5):578-596, 2009.

5. J. Bogaert, S. Dymarkowski, A.M. Taylor, and V. Muthurangu. Clinical cardiac MRI. Springer, 2012.

6. Yi Su, May-Ling SUI, Chi-Wan Lim, soo-Kng Teo, Ru-San tan, Min Wan, and Senthil Kumar Selvaraj. Automatic correction of motion artifacts in 4d left ventricle model reconstructed from mri. Computing in Cardiology 2014, 41:705-708., 2014.

7. A.G. Chandler, R.J. Pinder, T. Netsch, J.A. Schnabel, D.J Hawkes, D.L.G. Hill, and R. Razavi. Correction of misaligned slices in multi-slice cardiovascular magnetic resonance using slice-to-volume registration. Journal of Cardiovascular Magnetic Resonance, 10(1):1-9, 2008.

8. C. Zakkaroff, A. Radjenovic, J. Greenwood, and D. Magee. Stack alignment transform for misalignment correction in cardiac MR cine series. Technical report, University of Leeds, 2012.

9. J. Lotjonen, M. Pollari, S. Kivisto, and K. Lauerma. Correction of movement artifacts from 4-D cardiac short- and long-axis MR data. In International Conference on Medical Image Computing and Computer-Assisted Intervention, pages 405-412, 2004.

10. A.A. Goshtasby and D.A. Turner. Fusion of short-axis and long-axis cardiac MR images. In Mathematical Methods in Biomedical Image Analysis, 1996., Proceedings of the Workshop on, pages 202-211, Jun 1996.

11. K. McLeish, D.L.G. Hill, D. Atkinson, J.M. Blackall, and R. Razavi. A study of the motion and deformation of the heart due to respiration. IEEE Transactions on Medical Imaging, 21(9):1142-1150, 2002.

12. J.C. Bezdek and R.J. Hathaway. Convergence of alternating optimization. Neural, Parallel Sci. Comput., 11(4):351-368, 2003.

13. D. Perperidis. Spatio-temporal registration and modelling of the heart using cardiovascular MR imaging. PhD thesis, Imperial College London, 2006.

14. M.J. Ledesma-Carbayo, P. Kellman, A.E. Arai, and E.R. McVeigh. Motion corrected free-breathing delayed-enhancement imaging of myocardial infarction using nonrigid registration. Journal of Magnetic Resonance Imaging, 26(1):184-190, 2007.

15. P. Kovesi. Image features from phase congruency. Videre: A Journal of computer vision research, 1(3):1-26, 1999.

16. S. Bernstein, J.-L. Bouchot, M. Reinhardt, and B. Heise. Generalized analytic signals in image processing: comparison, theory and applications. In Quaternion and Clifford Fourier Transforms and Wavelets, pages 221-246. Springer, 2013.

17. C. Kelly, S. Neubauer, R. Choudhury, E. Dall'Armellina, and V. Grau. A local phase-based algorithm for registration of CMR scans from multiple visits. In Computing in Cardiology 2014, pages 937-940. IEEE, 2014.

18. K. G. Derpanis. Quadrature filters. Department of Computer Science and Engineering, York University, 2005. 